\title{
Association Between Urinary Phthalates and Pubertal Timing in Chinese Adolescents
}

\author{
Huijing Shi ${ }^{1}$, Yang $\mathrm{Cao}^{2}$, Qing Shen ${ }^{3}$, Yan Zhao ${ }^{1}$, Zhe Zhang ${ }^{1}$, and Yunhui Zhang ${ }^{1}$ \\ ${ }^{1}$ School of Public Health, Fudan University \& Key Laboratory of Public Health Safety, Chinese Ministry of Education, Shanghai, China \\ ${ }^{2}$ Division of Epidemiology, Institute of Environmental Medicine, Karolinska Institute, Stockholm, Sweden \\ ${ }^{3}$ Department of Public Health, Karolinska Institutet, Stockholm, Sweden
}

Received October 14, 2014; accepted March 12, 2015; released online July 25, 2015

Copyright (C) 2015 Huijing Shi et al. This is an open access article distributed under the terms of Creative Commons Attribution License, which permits unrestricted use, distribution, and reproduction in any medium, provided the original author and source are credited.

\begin{abstract}
Background: Phthalates are synthetic chemicals and ubiquitous environmental contaminants, with hormonal activity that may alter the course of pubertal development in children.

Objectives: To determine whether exposure to phthalate metabolites is associated with timing of pubertal development in a cross-sectional study of a school-based clustered sample of 503 children from a suburban district in Shanghai, China, who were 7-14 years of age at enrollment (2010 October to November).

Methods: We analyzed six phthalate metabolites in urine samples by isotope-dilution liquid chromatography tandem mass spectrometry. The associations of exposures to phthalates with pubertal timing of testes, breast, and pubic hair development (represented as Tanner stages) were evaluated using an ordered logistic regression model adjusted for chronological age, body fat proportion (BF\%), and parental education.

Results: In boys, urinary mono-n-butyl phthalate (MBP) levels were negatively associated with testicular volume, and mono (2-ethyl-5-hydroxyhexyl) phthalate (MEHHP) and mono (2-ethyl-5-oxohexyl) phthalate (MEOHP) levels were negatively associated with pubic hair stages. The odds of being in an advanced stage were decreased by 43\%-51\%. In girls, mono (2-ethylhexyl) phthalate (MEHP), MEHHP, and MEOHP levels, as well as the sum of these levels, were positively associated with breast stages, and the association was much stronger in girls with high $\mathrm{BF} \%$; the odds of being in an advanced stage were increase by $29 \%$ to $50 \%$.

Conclusions: Phthalate metabolites investigated in this study show significant associations with pubertal timing both in boys and in girls, especially among girls with high $\mathrm{BF} \%$.
\end{abstract}

Key words: phthalate; sexual development; puberty onset; adolescent; body fat

\section{INTRODUCTION}

Puberty entails an individual's transition period from a nonreproductive to a reproductive state and is characterized by rapid physiological changes. The onset of puberty varies by 4 to 5 years among normal boys and girls. ${ }^{1}$ Over the past 50 years, a trend toward earlier age at onset of puberty, especially in girls, has been reported. ${ }^{2-6}$ At the population level, a secular trend in the timing of puberty may influence behavioral disorders and adult health, which may lead to adverse social and medical conditions. ${ }^{7,8}$ Although some have thought this trend to be due to improvements in general health and nutrition, ${ }^{9}$ there has also been considerable concern that hormonally active substances, called endocrine-disrupting chemicals (EDCs), are involved in alterations of the onset and progression of pubertal development. ${ }^{10,11}$ EDCs have been implicated in numerous physiological processes affecting normal reproductive health in human beings and animals. ${ }^{12}$ Specific EDCs that behave like estradiol, such as bisphenol A, may act as hormone agonists and accelerate pubertal development in animal models. However, some EDCs, including phthalates, have both agonist and antagonist effects in animals; these varying effects are likely related to alternative mechanism, dose levels, and exposure timing. ${ }^{13}$

Phthalates are widely used in personal care and consumer products, including children's toys and medical devices, to make them soft and flexible, as well as in cosmetics, where phthalates act as a vehicle for fragrance. ${ }^{14,15}$ Humans can be 
exposed to phthalates through inhalation, ingestion, dermal exposure, and medical treatments. ${ }^{16,17}$ Previous studies have mainly focused on their adverse effects on male reproductive development. ${ }^{18-20}$ Recently, human epidemiological studies of the association between phthalate exposure and pubertal development have caused concern. In boys, phthalate exposure has been associated with earlier age at pubarche ${ }^{21}$ and pubertal gynecomastia. ${ }^{22}$ High phthalate exposure has also been associated with changes in pubertal timing in girls, ${ }^{23-26}$ although this association is controversial. ${ }^{21,27}$

The effects of phthalates on the timing of puberty, while subtle, may have significant public health implications. A population shift in pubertal timing may confer health risks for later disease, both physical (eg, adult obesity and height loss) and psychological (eg, delinquency, substance use, and risky sexual behavior). Altered onset of puberty and growth in children has been considered a problematic issue in many countries. ${ }^{28-30}$ However, given the inconsistent results existing among girls and the paucity of evidence in boys, further investigation of the relationship between phthalates exposure and pubertal development in children is urgently needed.

Based on a large cross-sectional survey on pubertal timing and health effects in China, we analyzed urinary phthalate concentrations in a random sample and measured the pubertal stage of children. We aimed to provide further insight into the possible role of phthalate exposure in the timing of pubertal development during a critical time window in children. The hypothesis of our study was that high urinary phthalate concentrations were positively associated with relatively earlier development of pubertal indices in girls but negatively associated with the similar indices in boys.

\section{MATERIALS AND METHODS}

\section{Study population}

After the multi-centered Chinese Puberty Research Collaboration was initiated in September 2010, a large cross-sectional survey-the Pubertal Timing and Health Effects in Chinese Children (PTHEC) study-was conducted in eight cities from October to November $2010 .^{30,31}$ Shanghai was one of the cities involved in the PTHEC study. By a stratified multistage cluster sampling method, one urban district and one suburban district were selected from eight central urban districts and nine suburban districts in Shanghai. After excluding students with congenital malformations and genetic, metabolic, or chronic endocrine diseases, 3462 students (1787 boys and 1675 girls) aged 6 to 18 years were invited to join the study, which included anthropometric measurement, sexual maturation assessment, and a questionnaire interview. Of these, primary school students in grades three through seven from one suburban district were selected for further laboratory analysis of urine samples. The study was approved by the Ethics Review Committee of Fudan University (IRB\#2010-11-0242; 2011-
03-0280). Informed consent was explained to all children and their parents, and signed informed consent forms were voluntarily obtained from the parents before participation.

\section{Data collection}

A set of questionnaires was completed by the students and their guardians, which included perinatal factors, demographic variables, perceived physical growth and development, emotion and feeling, physical activities, parental information, sleeping and study habits, dietary habits and intake, and experience of spermarche (in boys) or menarche (in girls).

\section{Physical examination}

Anthropometric measures, including body weight, height, body mass index (BMI), and tricep and subscapular skinfold thicknesses, were measured by physical examination according to WHO-recommended methods and protocols at the time when the urine phthalate samples were collected in all children. ${ }^{32}$ The equipment was calibrated daily using the manufacturer's calibrator. Body fat proportion (BF\%) was calculated using Yao's formula, which is widely used in Chinese school-age children aged 7-12 years. ${ }^{33}$ The formulae were $\mathrm{BF} \%=6.931+0.428 \mathrm{X}$ and $\mathrm{BF} \%=7.896+0.458 \mathrm{X}$ for boys and girls, respectively, where $\mathrm{X}$ is the sum of tricep and subscapular skin fold thickness in millimeters.

The sexual maturity of testes (in boys), breasts (in girls), ${ }^{34}$ and pubic hair (in both boys and girls) was assessed privately by a male urologist (for boys) or a female pediatrician (for girls). Testicular volume (TV) was estimated by palpation to the nearest whole milliliter using Prader's orchidometer and divided into four levels (T1-T4) as $<4 \mathrm{~mL}, 4-11 \mathrm{~mL}$, $12-19 \mathrm{~mL}$, and $\geq 20 \mathrm{~mL}^{11,34,35}$ In cases of discrepancy between the left and right side, the largest measurement was used for classification. Sexual maturity stages from stage 1 (indicating immaturity) to stage 5 (indicating full maturity) of breasts (B1-B5) and pubic hair (PH1-PH5) were assessed by inspection and palpation according to the methods of Marshall \& Tanner. ${ }^{36,37}$ The students were asked whether or not they had had their first nocturnal emission (for boys) or first menstrual bleeding (for girls).

\section{Urinary biomarker measurement}

Spot urinary samples were collected from each student on the day of physical examination. All specimens were collected with glass devices to avoid contamination and stored at $-20^{\circ} \mathrm{C}$ until analysis. Six phthalate metabolites were measured: mono-n-butyl phthalate (MBP), mono-methyl phthalate (MMP), monoethyl phthalate (MEP), mono (2-ethylhexyl) phthalate (MEHP), mono (2-ethyl-5-hydroxyhexyl) phthalate (MEHHP), and mono (2-ethyl-5-oxohexyl) phthalate (MEOHP). The sum of MEHP, MEHHP, and MEOHP concentrations was represented as $\Sigma$ MEHP. Phthalate metabolites in urine extract were resolved using an Agilent 1100 Series high-performance liquid chromatography system 
(Agilent Technologies, Santa Clara, CA, USA) and detected with an API 2000 electrospray triple quadrupole mass spectrometer (Applied Biosystems, Foster City, CA, USA). ${ }^{13}$ $\mathrm{C}_{4}$-labeled internal standards and conjugated internal standards were used to increase the precision of the measurements. Analysts at the Key Laboratory of Public Health Safety, who performed the tests for the present study, were blinded to all information concerning our subjects. For concentrations below the limits of detection (LODs), corresponding to $0.25 \mu \mathrm{g} / \mathrm{L}$ (MMP) and $0.50 \mu \mathrm{g} / \mathrm{L}$ (MBP, MEP, MEHP, MEOHP, and MEHHP), we used an imputed value equal to one-half the LOD.

We used specific gravity (SG) to correct for urinary dilution, as recommended by Hauser et al. ${ }^{15}$ SG was measured using a handheld refractometer (PAL10-S; Atago, Tokyo, Japan). The correction formula was $\mathrm{Pc}=\mathrm{P} \times$ $(1.024-1) /(\mathrm{SG}-1)$, where $\mathrm{Pc}$ is the specific gravitycorrected phthalate metabolite concentration $(\mu \mathrm{g} / \mathrm{L})$ and $\mathrm{P}$ is the experimental phthalate metabolite concentration.

\section{Statistical methods}

Arithmetic mean and standard deviation (SD) of age, height, weight, and $\mathrm{BF} \%$, and geometric mean (GM) and $95 \%$ confidence interval (CI) of SG-corrected urine phthalate metabolite concentrations were separately calculated for boys and girls. The differences in demographic characteristics and phthalate metabolite levels between boys and girls were tested using a $t$-test or Wilcoxon's rank-sum test. The correlations between phthalate metabolite levels and covariates were measured using Spearman correlation coefficient $r_{s}$.

To evaluate the association of phthalate metabolite levels with pubertal timing, an ordered logistic regression model was constructed to assess the association of sexual maturation of $\mathrm{TV}$, breasts, or pubic hair and SG-corrected urine phthalate metabolite concentrations, adjusting for pre-determined covariates, including chronological age (the number of the months after birth divided by 12 ), $\mathrm{BF} \%$, and parental education. The purpose of this analysis was to compare the pubertal development stage of an adolescent in relation to peers of the same age and $\mathrm{BF} \%$. That is, at a given age and $\mathrm{BF} \%$, a higher sexual development stage signifies relatively earlier pubertal timing. Because parental education is an important proxy of socioeconomic status of a family and has close relationship with child health and puberty, we included it as a covariate in the model. ${ }^{38,39}$

Because the SG-corrected phthalate concentrations were approximately log-normally distributed, a natural $\log$ transformation was applied to normalize the data. In ordered logistic regression, the predicted probabilities for each TV level and breast or pubic hair stage at different phthalate concentrations were calculated and plotted to illustrate the trend of these probabilities with increased phthalate concentrations.
Because adipose tissue is a source of pubertal hormones, ${ }^{40}$ we also investigated modification of biomarker associations by $\mathrm{BF} \%$. We divided the $\mathrm{BF} \%$ into low $\mathrm{BF}(\mathrm{BF} \% \leq 20 \%$ in boys or $\leq 25 \%$ in girls) and high $\mathrm{BF}$ (BF\% $>20 \%$ in boys or $>25 \%$ in girls) groups and phthalate concentrations into low exposure ( $\leq$ median concentration) and high exposure ( $>$ median concentration) groups, then introduced an interaction term of $\mathrm{BF}$ and exposure in the model.

For multivariate analysis, the list-wise deletion method was used for handling missing data, and students were excluded from analyses if any single value for dependent or independent variables was missing. ${ }^{41}$ The difference in demographic characteristics between excluded and included students was compared using the $t$ test or Chi-square test.

To assess the robustness of our results to various methodological decisions, we conducted several sensitivity analyses. First, we defined phthalate concentrations as continuous and ordinal variables with two, three, four, and five categories divided by median, tertiles, quartiles, and quintiles, respectively. Second, we fitted univariate models separately for continuous and ordinal phthalate concentration variables to estimate the crude odds ratios (ORs). Third, we introduced other covariates in the univariate models of continuous and ordinal phthalate concentration variables to estimate the adjusted ORs. The goodness of fit of different modeling strategies and ORs was compared across the models.

All tests were two-sided, and a $P$-value of less than 0.05 was considered statistically significant. All statistical analyses were performed with the Stata software version 12.1 (StataCorp, College Station, TX, USA).

\section{RESULTS}

Ultimately, 503 primary-school students (252 boys and 251 girls) from a suburban district in Shanghai were selected for urine sample analysis. The detailed flow diagram of recruitment is shown in Figure 1. Due to lack of a qualified urine sample, eight children were excluded from laboratory testing. All studied phthalate metabolites could be detected in all analyzed samples, and the detection rates of monophthalates were all higher than $95 \%$, except for MEP and MEHP.

For demographics and exposure characteristics, boys had significantly higher weight and $\mathrm{BF} \%$, as well as higher exposure to MMP, MEOHP, and $\Sigma$ MEHP than girls (Table 1). Over $60 \%$ of boys had high BF and over $85 \%$ of girls had low BF. The proportion of boys with high phthalate exposure and high BF was over $30 \%$. However, over $40 \%$ of girls had low $\mathrm{BF}$ and low phthalate exposure, except for exposure to MEHP (see eTable 1). In all children, age at enrollment and parental education were correlated with concentrations of some phthalates, but the relationship was weak $\left(\left|r_{s}\right|<0.26\right.$ and $\left|r_{s}\right|<0.18$ for age and parental education, respectively). 


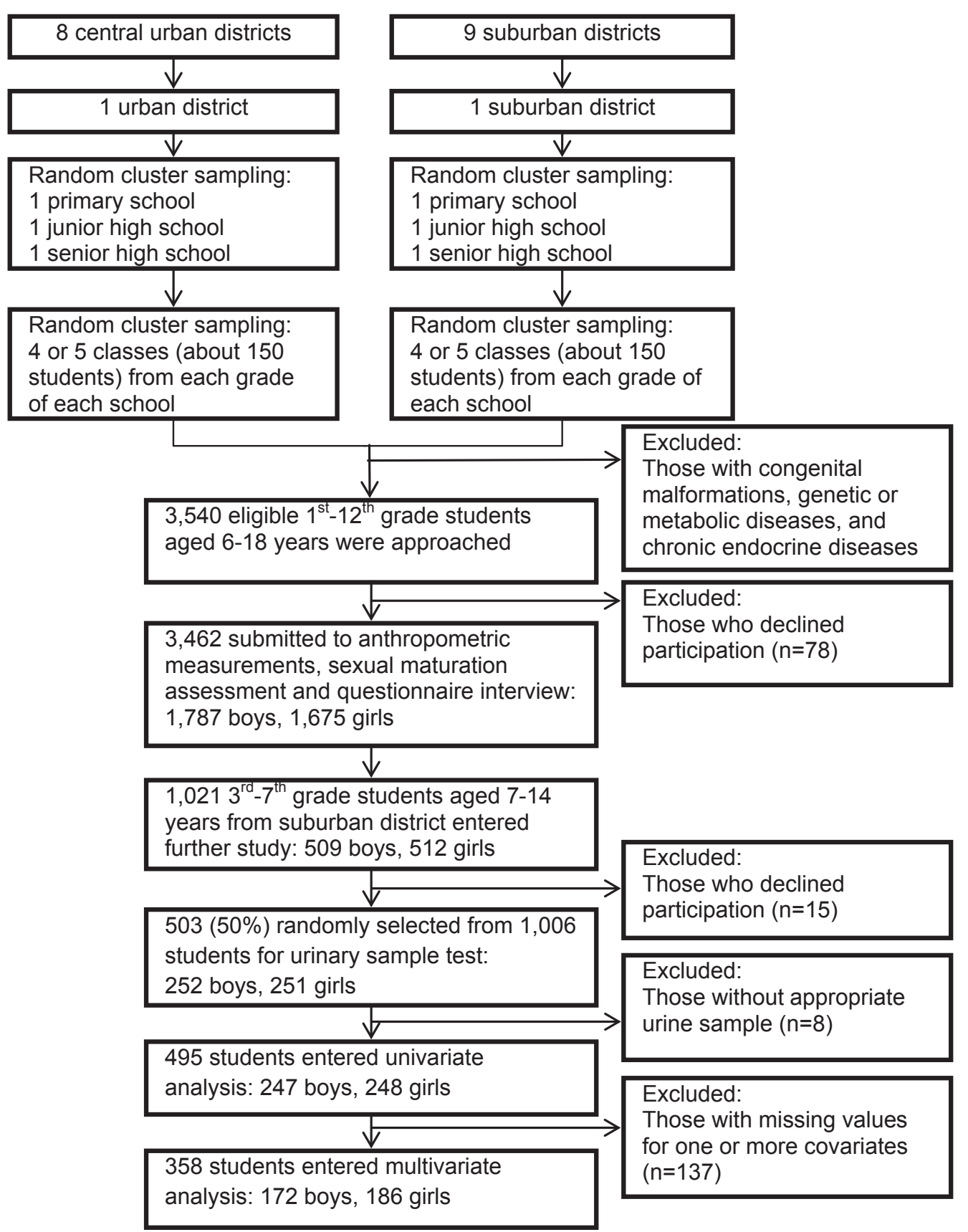

Figure 1. Flow chart of participant recruitment.

Except for a weak positive correlation between BF\% and MEP concentration $\left(r_{s}=0.11, P=0.035\right)$, there were no significant correlations between $\mathrm{BF} \%$ and concentration of other phthalate metabolites.

The crude ORs derived from univariate ordered logistic regression models show that TV in boys, breast stage in girls, and pubic hair stage in boys and girls were all negatively associated with concentrations of two to four non-MEP phthalate metabolites (data not shown). MEP level was positively associated with increased TV in boys and earlier menarche in girls, and the risk of presenting with larger TV and earlier menarche than age- and $\mathrm{BF} \%$-matched peers were increased by $18 \%$ and $33 \%$, respectively.
Due to missing data on covariates, 137 children were excluded from multivariate ordered logistic analysis. Analyses revealed no significant differences between excluded and included children in demographic characteristics and phthalate concentrations. After adjusting for chronological age, BF\%, and parental education, MBP level was negatively associated with TV, and MEHHP and MEOHP levels were negatively associated with pubic hair stages in boys. The risk of being in advanced stages was decreased by $43 \%-51 \%$ compared to age- and $\mathrm{BF} \%$-matched peers. The association between phthalate levels and breast stage in girls was reversed after adjusting for covariates. MEHP, MEHHP, MEOHP, and ¿MEHP were positively associated with breast stage, and 
Table 1. Demographic and exposure characteristics

\begin{tabular}{|c|c|c|}
\hline & Boys $(n=252)$ & Girls $(n=251)$ \\
\hline Mean (SD) age, years & $10.7(1.5)$ & $10.7(1.5)$ \\
\hline \multicolumn{3}{|l|}{ Father's education, $n(\%)$} \\
\hline Primary or lower & $1(0.55)$ & $3(1.60)$ \\
\hline Junior high school & $49(26.78)$ & 37 (19.79) \\
\hline Senior high school & $76(41.53)$ & $85(45.45)$ \\
\hline College or university & $50(27.32)$ & $59(31.55)$ \\
\hline Graduate education & $7(3.83)$ & $3(1.60)$ \\
\hline \multicolumn{3}{|l|}{ Mother's education, $n(\%)$} \\
\hline Primary or lower & $4(2.20)$ & $2(1.07)$ \\
\hline Junior high school & $43(23.63)$ & $38(20.32)$ \\
\hline Senior high school & $71(39.01)$ & $85(45.45)$ \\
\hline College or university & $59(32.42)$ & $57(30.48)$ \\
\hline Graduate education & $5(2.75)$ & $5(2.67)$ \\
\hline Mean (SD) height, $\mathrm{cm}$ & $146.1(11.5)$ & $145.1(10.9)$ \\
\hline Mean (SD) weight, $\mathrm{kg}^{\mathrm{a}}$ & $43.8(14.3)$ & $39.2(11.5)$ \\
\hline Mean (SD) body fat composition, $\%^{a}$ & $24.9(9.2)$ & $17.7(7.1)$ \\
\hline \multicolumn{3}{|l|}{ Boy's testicular volume, $n(\%)$} \\
\hline$<4 \mathrm{~mL}$ & $137(54.37)$ & \\
\hline $4-11 \mathrm{~mL}$ & $72(28.57)$ & \\
\hline $12-19 \mathrm{~mL}$ & $25(9.92)$ & \\
\hline$\geq 20 \mathrm{~mL}$ & $18(7.14)$ & \\
\hline \multicolumn{3}{|l|}{ Tanner stage of girls' breast, $n(\%)$} \\
\hline B1 & & $55(21.91)$ \\
\hline B2 & & $74(29.48)$ \\
\hline B3 & & $64(25.50)$ \\
\hline B4 & & $46(18.33)$ \\
\hline B5 & & $12(4.78)$ \\
\hline \multicolumn{3}{|l|}{ Tanner stage of pubic hair, $n(\%)^{b}$} \\
\hline $\mathrm{PH} 1$ & $219(86.90)$ & $175(69.72)$ \\
\hline $\mathrm{PH} 2$ & $18(7.14)$ & $27(10.76)$ \\
\hline $\mathrm{PH} 3$ & $12(4.76)$ & $41(16.33)$ \\
\hline $\mathrm{PH} 4$ & $3(1.19)$ & $7(2.79)$ \\
\hline PH5 & - & $1(0.40)$ \\
\hline \multicolumn{3}{|l|}{ Boy's spermarche/girl's menarche, $n(\%)$} \\
\hline Yes & $8(4.17)$ & $51(25.89)$ \\
\hline No & $184(95.83)$ & $146(74.11)$ \\
\hline \multicolumn{3}{|c|}{ Urinary specific gravity-adjusted phthalate concentrations, $\mu \mathrm{g} / \mathrm{L}, \mathrm{GM}(95 \% \mathrm{Cl})^{\mathrm{c}}$} \\
\hline MBP & $28.57(25.98,31.41)$ & $19.60(16.35,23.50)$ \\
\hline MMP* & $14.18(12.83,15.67)$ & $11.50(10.28,12.87)$ \\
\hline MEP & $1.15(0.93,1.41)$ & $1.14(0.91,1.43)$ \\
\hline MEHP & $1.65(1.40,1.94)$ & $1.65(1.41,1.92)$ \\
\hline MEHHP & $16.10(14.65,17.68)$ & $14.5(12.97,16.22)$ \\
\hline MEOHP* & $5.77(5.25,6.37)$ & $4.72(4.18,5.33)$ \\
\hline$\Sigma \mathrm{MEHP} *$ & $24.49(22.31,26.89)$ & $21.52(19.21,24.11)$ \\
\hline
\end{tabular}

$\mathrm{Cl}$, confidence interval; GM, geometric mean; MBP, monobutyl phthalate; MEHHP, mono-(2-ethyl-5-hydroxyhexyl) phthalate; MEHP, mono-(2-ethylhexyl) phthalate; MEOHP, mono-(2-ethyl-5-oxohexyl) phthalate; MEP, mono-ethyl phthalate; MMP, mono-methyl phthalate; IMEHP, sum of MEHP, MEHHP, and MEOHP concentrations. a Significant difference between boys and girls, $P<0.05, t$ test. bSignificant difference between boys and girls, $P<0.05$, Wilcoxon rank-sum test.

'Values based on test results for 247 boys and 248 girls.

the risk of being in advanced stages was increase by $29 \%$ to $50 \%$ compared to age- and BF\%-matched peers (Table 2). No association was found between phthalate levels and spermarche in boys or pubic hair stage and menarche in girls.

The predicted probabilities of having larger TV and higher pubic hair stage in boys decreased with MBP, MEHHP, and MEOHP concentrations (data not shown). The predicted probabilities of presenting with lower breast stage were decreased, and predicted probabilities of presenting higher breast stage were increased, with MEHP, MEHHP, MEOHP, and $\Sigma$ MEHP concentrations, and this was most apparent in $\Sigma$ MEHP for older girls (Figure 2).

Although no statistically significant interactions were found between $\mathrm{BF} \%$ and phthalate levels, we did find that the
Table 2. Odds ratios and $95 \%$ confidence intervals ${ }^{a}$ of presenting higher versus lower pubertal development levels per 1-unit increase in logarithmic urine specific gravity-adjusted phthalate concentrations

\begin{tabular}{|c|c|c|c|}
\hline Boys & $\begin{array}{l}\text { Testicle volume } \\
\qquad(n=176)\end{array}$ & $\begin{array}{c}\text { Pubic hair } \\
(n=176)\end{array}$ & $\begin{array}{l}\text { First nocturnal emission } \\
\qquad(n=170)\end{array}$ \\
\hline MBP & $0.58(0.34,0.99)^{*}$ & $0.91(0.45,1.83)$ & $1.31(0.43,3.98)$ \\
\hline MMP & $1.46(0.94,2.26)$ & $0.74(0.43,1.27)$ & $1.38(0.52,3.66)$ \\
\hline MEP & $1.10(0.90,1.35)$ & $1.15(0.89,1.48)$ & $0.87(0.53,1.41)$ \\
\hline MEHP & $1.23(0.94,1.62)$ & $0.87(0.61,1.25)$ & $0.77(0.42,1.43)$ \\
\hline MEHHP & $0.68(0.41,1.11)$ & $0.52(0.28,0.97)^{*}$ & $1.05(0.36,3.05)$ \\
\hline MEOHP & $0.72(0.44,1.15)$ & $0.49(0.26,0.91)^{*}$ & $0.93(0.32,2.70)$ \\
\hline$\Sigma \mathrm{MEHP}$ & $0.82(0.51,1.33)$ & $0.57(0.30,1.07)$ & $1.14(0.40,3.29)$ \\
\hline Girls & $\begin{array}{c}\text { Breast } \\
(n=182)\end{array}$ & $\begin{array}{l}\text { Pubic hair } \\
(n=182)\end{array}$ & $\begin{array}{l}\text { Menarche } \\
(n=178)\end{array}$ \\
\hline MBP & $1.13(0.94,1.35)$ & $1.10(0.87,1.40)$ & $0.91(0.68,1.23)$ \\
\hline MMP & $0.83(0.59,1.15)$ & $0.96(0.62,1.50)$ & $1.10(0.61,1.97)$ \\
\hline MEP & $1.00(0.86,1.17)$ & $1.08(0.90,1.31)$ & $1.11(0.89,1.39)$ \\
\hline MEHP & $1.29(1.01,1.64)^{*}$ & $1.11(0.82,1.50)$ & $0.95(0.64,1.41)$ \\
\hline MEHHP & $1.45(1.06,1.98)^{*}$ & $1.00(0.68,1.46)$ & $0.72(0.44,1.19)$ \\
\hline MEOHP & $1.46(1.09,1.95)^{*}$ & $1.09(0.77,1.56)$ & $0.75(0.48,1.20)$ \\
\hline$\Sigma \mathrm{MEHP}$ & $1.50(1.10,2.04)^{*}$ & $1.05(0.72,1.54)$ & $0.74(0.45,1.23)$ \\
\hline
\end{tabular}

MBP, monobutyl phthalate; MEHHP, mono-(2-ethyl-5-hydroxyhexyl) phthalate; MEHP, mono-(2-ethylhexyl) phthalate; MEOHP, mono-(2ethyl-5-oxohexyl) phthalate; MEP, mono-ethyl phthalate; MMP, monomethyl phthalate; $\Sigma$ MEHP, sum of MEHP, MEHHP, and MEOHP concentrations.

${ }^{a}$ Adjusted for chronological age, body fat composition and parental education.

$* P<0.05$.

associations of phthalate concentrations with breast development were much stronger in girls with high $\mathrm{BF} \%$. Compared with girls with high phthalate levels and low $\mathrm{BF} \%$, the ORs of presenting with higher breast stage in girls with high phthalate levels and high $\mathrm{BF} \%$ were 3.52 versus $1.31,6.99$ versus 1.16 , 7.71 versus 1.42 , and 7.83 versus 1.50 for MEHP, MEHHP, MEOHP, and $\Sigma$ MEHP, respectively (Table 3).

\section{DISCUSSION}

In a cross-sectional analysis of a subgroup of the large multicentered PTHEC study, we examined the associations between concurrent exposure to phthalates, which are known to possess hormonal activity, and pubertal development. The detection rates of the examined urinary phthalate metabolites were all over $80 \%$, which indicated that exposure to phthalates was a routine occurrence for children. The average concentrations of phthalate metabolites ranged from to 1.14 to $28.57 \mu \mathrm{g} / \mathrm{L}$. These levels were comparable with those reported by Wang et $\mathrm{al}^{42}$; however, urinary concentrations of phthalate metabolites in our study were significantly higher in boys than in girls.

Consistent with our a priori hypothesis, positive trends were observed for the association of phthalate biomarkers with breast development in females. Specifically, MEHP, MEHHP, MEOHP, and $\mathrm{MEHP}$ were found to be associated with $29 \%$ to $50 \%$ increases in the risk of being in an advanced breast stages at a given age. These results were consistent 

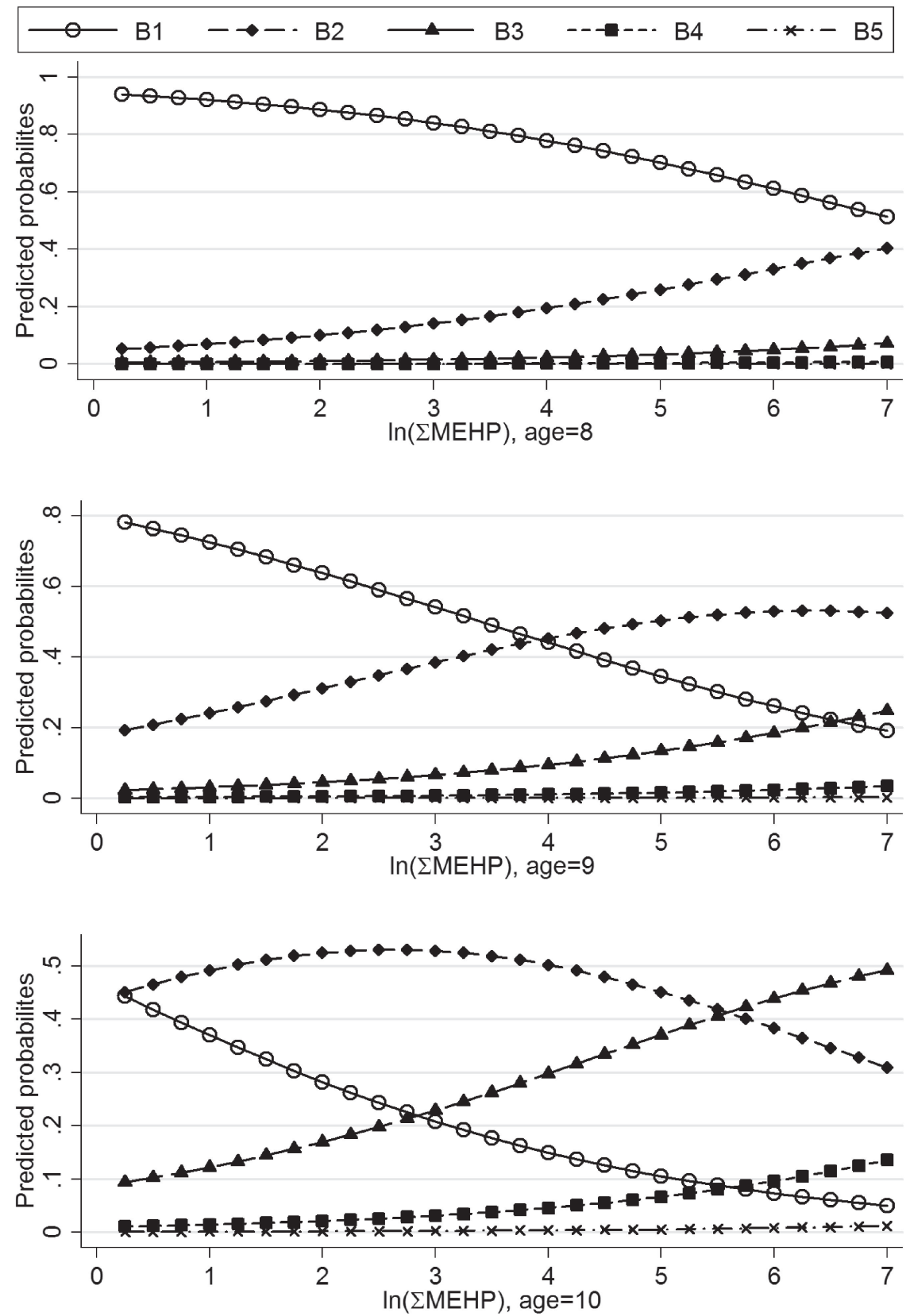

Figure 2. Predicted probabilities of breast stages (B1-B5) of girls $(n=182)$ with $\Sigma$ MEHP concentrations at ages 8 , 9 , and 10 years.

with previous findings, ${ }^{23,24,43}$ which suggested that high phthalate exposure might be one cause of premature breast development. However, in one American study, Lomenick et al reported no difference in phthalate exposure in girls with and without precocious puberty. ${ }^{25}$ In several Danish studies, Frederiksen et al did not find any association between phthalate exposure and breast development. ${ }^{21,26}$ Furthermore, in a study by Wolff et al, low-molecular-weight phthalate biomarkers were found to have a weak positive association with breast stage, while high-molecular-weight phthalate metabolites had no association. ${ }^{22}$ These discrepancies might be due to differences in the levels of phthalates measured in different studies. The subjects in our study were from a randomized cluster sample with an age range (from pre- puberty to the early and middle periods of puberty) that involves relatively larger interpersonal variation in breast development timing, which increased the representativeness of the samples and might more comprehensively and sensitively reflect the association between environmental exposure and varying pubertal timing. The inverse association found between phthalate exposure and girls' breast development, even after adjusting for confounding variables, may be due to the interaction between phthalate metabolites and body fat. Because of the liposolubility of phthalates, the effect of phthalates on breast development would be more evident in girls with more body fat, who might have greater phthalate accumulation in the body; such a relationship was confirmed in the interaction analysis. 
Table 3. Association of breast development with interaction between body fat ${ }^{\mathrm{a}}$ and phthalate concentrations ${ }^{\mathrm{b}}$ in girls

\begin{tabular}{lccc}
\hline Exposures & OR & $95 \% \mathrm{Cl}$ & $P^{\mathrm{c}}$ \\
\hline MEHP & & & \\
low PC, Low BF & Reference & & \\
low PC, High BF & 2.76 & $1.16,6.60$ & 0.022 \\
high PC, Low BF & 1.31 & $0.71,2.41$ & 0.391 \\
high PC, High BF & 3.52 & $0.45,27.48$ & 0.231 \\
\hline MEHHP & & & \\
low PC, Low BF & Reference & & \\
low PC, High BF & 2.30 & $0.97,5.45$ & 0.058 \\
high PC, Low BF & 1.16 & $0.63,2.14$ & 0.624 \\
high PC, High BF & 6.99 & $1.03,47.29$ & 0.046 \\
\hline MEOHP & & & \\
low PC, Low BF & Reference & & \\
low PC, High BF & 2.51 & $1.06,5.99$ & 0.037 \\
high PC, Low BF & 1.42 & $0.78,2.61$ & 0.254 \\
high PC, High BF & 7.71 & $1.13,52.52$ & 0.037 \\
\hline IMEHP & & & \\
low PC, Low BF & Reference & & \\
low PC, High BF & 2.57 & $1.08,6.10$ & 0.033 \\
high PC, Low BF & 1.50 & $0.81,2.75$ & 0.195 \\
high PC, High BF & 7.83 & $1.15,53.32$ & 0.035 \\
\hline BF
\end{tabular}

$\mathrm{BF}$, body fat; $\mathrm{Cl}$, confidence interval; MEHHP, mono-(2-ethyl-5hydroxyhexyl) phthalate; MEHP, mono-(2-ethylhexyl) phthalate; MEOHP, mono-(2-ethyl-5-oxohexyl) phthalate; OR, odds ratio; PC, phthalate concentration; $\Sigma \mathrm{MEHP}$, sum of MEHP, MEHHP, and MEOHP concentrations.

${ }^{a}$ Divided into low and high groups by body fat composition $\leq 25 \%$ and $>25 \%$ for girls.

${ }^{b}$ Divided into low exposure and high exposure groups by $\leq$ median and >median of specific phthalate concentrations of all children.

${ }^{\mathrm{c}}$ Adjusted for chronological age and parental education.

The effect of phthalate exposure on male pubertal development has been sparsely evaluated. Mieritz et al did not find any association between current phthalate exposure and pubertal timing, ${ }^{44}$ while higher di-n-butyl phthalate exposure was found to be associated with earlier age at pubarche in another study. ${ }^{26}$ However, in the present study, MBP was found to be associated with a $42 \%$ decrease in the risk of larger TV, and MEHHP and MEOHP were found to be associated with $48 \%$ to $51 \%$ decreases in the risk of being in advanced pubic hair stages, even after controlling for some potential confounders. Our results suggest negative associations between phthalate exposure and pubertal development in males.

Pubertal onset and progression is primarily regulated by the endocrine system through chemical messengers, specifically the sexual hormones. Both animal and human findings suggest that phthalates have antiandrogenic properties. ${ }^{45-48}$ In males, androgen levels determine the onset of testicular growth and pubic hair development, so we thought that delayed pubertal development in males in our study might be attributed to the antiandrogenic effects of phthalates. However, one of the etiologic explanations for advanced breast development in females is increased estrogenic sensitivity or estrogen- androgen ratio in breast tissue, or both. As in vivo and in vitro studies have indicated negligible estrogenic activity for DEHP, ${ }^{49}$ we speculated that the anti-androgenic effects of DEHP metabolites (MEHP, MEHHP, and MEOHP) resulted in altered estrogen-androgen balance and influenced breast tissue responsiveness to estrogen.

Phthalates, which are considered environmental obesogens, tend to accumulate in fat tissues. Phthalate exposure might contribute to adipogenesis and induce obesity. Low levels of MEHP have been shown to promote adipocyte differentiation in a dose-dependent manner in mice. ${ }^{50}$ Urinary phthalate levels have also been shown to be associated with body size indices in humans. ${ }^{42}$ In the present study, although no statistically significant interactions were found between $\mathrm{BF} \%$ and phthalate concentrations, the association between phthalate levels and breast development was found to be much stronger in girls with high $\mathrm{BF} \%$, which indicates the complexity of phthalates' impact on adipose tissue biology, hormone systems, and the central hypothalamic-pituitarygonadal axis, than in those with low $\mathrm{BF} \% .^{25,26,50}$ Follow-up studies on sex hormone levels and pubertal timing are needed to further explore the interactions among phthalate exposure, $\mathrm{BF} \%$, and puberty onset in children.

The sensitivity analyses indicated that, when we included the phthalate concentrations as ordinal variables in the model, the association between phthalate levels and pubertal development indices disappeared or reversed with decreased ordinal categories of phthalate concentrations. The most consistent results were found between the models using five-category ordinal phthalate concentrations and the models using continuous phthalate concentrations, which suggests that treating phthalate concentrations as continuous variables would be more suitable for risk assessment purposes.

There are some limitations to our study. First, we used spot urine samples to assess each subject's phthalate levels. Due to the short half-lives of phthalate metabolites, a single measurement of spot urine samples might not perfectly represent long-term exposure. Second, we did not measure circulatory levels of sex hormones. Although associations between phthalate concentrations and pubertal timing both in boys and in girls were found in the current study, we could not determine whether or not the associations are the result of hormonal activities of phthalate metabolites. Third, pubertal timing could determine some behaviors associated with phthalate exposures, such as eating packaged foods. Although we investigated dietary habits and intakes in the questionnaire, diet and behavior bias could not be excluded. Furthermore, the potential confounding effects of birth outcomes, living environment in young childhood, or exposure to other chemicals, especially chemicals with direct or indirect antiandrogenic properties, is unclear and cannot be accurately assessed.

To our knowledge, this is the largest study to date to investigate the association between phthalate concentrations 
and pubertal development in a community sample of primary-school students. In addition, our study used a detailed questionnaire survey and physical examination by trained specialists, which minimized the potential for misclassification. Furthermore, ordered logistic regression, an extension of logistic regression, was used in our study, which is particularly appropriate to investigate the relationship of ordered outcomes with demographic characteristics, biochemistry data, and self-assessment of health. The results from ordered logistics regression models can be more valid and sometimes more informative than those of ordinary leastsquares regression models when the distribution of outcome is highly non-normal. ${ }^{51,52}$

In conclusion, we found significant associations between urinary phthalate metabolite concentrations and pubertal stages in children aged 7 to 14 years. High phthalate concentrations were associated with delayed pubertal development in boys and advanced pubertal development in girls, which might reflect the antiandrogenic action of phthalates.

\section{ONLINE ONLY MATERIAL}

eTable 1. Distribution of low and high body fat $(\mathrm{BF})^{\mathrm{a}}$, and low and high phthalate exposure.

\section{ACKNOWLEDGEMENTS}

This research was supported by grants from the Natural Science Foundation of China (Grant 81072263, to Y.Z; Grant 81172684 , to H.S) and by an award from the Shanghai Municipal Health Bureau (Grant Number 12GWZX0301, to X.Q).

Conflicts of interest: None declared.

\section{REFERENCES}

1. Golub MS, Collman GW, Foster PM, Kimmel CA, Rajpert-De Meyts E, Reiter EO, et al. Public health implications of altered puberty timing. Pediatrics. 2008;121 Suppl 3:S218-30.

2. Herman-Giddens ME, Slora EJ, Wasserman RC, Bourdony CJ, Bhapkar MV, Koch GG, et al. Secondary sexual characteristics and menses in young girls seen in office practice: a study from the Pediatric Research in Office Settings network. Pediatrics. 1997;99:505-12.

3. Anderson SE, Dallal GE, Must A. Relative weight and race influence average age at menarche: results from two nationally representative surveys of US girls studied 25 years apart. Pediatrics. 2003;111:844-50.

4. Freedman DS, Khan LK, Serdula MK, Dietz WH, Srinivasan SR, Berenson GS. Relation of age at menarche to race, time period, and anthropometric dimensions: the Bogalusa Heart Study. Pediatrics. 2002;110:e43.

5. Aksglaede L, Sørensen K, Petersen JH, Skakkebaek NE, Juul A. Recent decline in age at breast development: the Copenhagen
Puberty Study. Pediatrics. 2009;123:e932-9.

6. Parent AS, Teilmann G, Juul A, Skakkebaek NE, Toppari J, Bourguignon JP. The timing of normal puberty and the age limits of sexual precocity: variations around the world, secular trends, and changes after migration. Endocr Rev. 2003;24: 668-93.

7. Patton GC, Hemphill SA, Beyers JM, Bond L, Toumbourou JW, McMorris BJ, et al. Pubertal stage and deliberate self-harm in adolescents. J Am Acad Child Adolesc Psychiatry. 2007;46: 508-14.

8. Schoeters G, Den Hond E, Dhooge W, van Larebeke N, Leijs M. Endocrine disruptors and abnormalities of pubertal development. Basic Clin Pharmacol Toxicol. 2008;102:168-75.

9. Euling SY, Herman-Giddens ME, Lee PA, Selevan SG, Juul A, Sørensen TI, et al. Examination of US puberty-timing data from 1940 to 1994 for secular trends: panel findings. Pediatrics. 2008;121 Suppl 3:S172-91.

10. Jacobson-Dickman E, Lee MM. The influence of endocrine disruptors on pubertal timing. Curr Opin Endocrinol Diabetes Obes. 2009;16:25-30.

11. Buck Louis GM, Gray LE Jr, Marcus M, Ojeda SR, Pescovitz $\mathrm{OH}$, Witchel SF, et al. Environmental factors and puberty timing: expert panel research needs. Pediatrics. 2008;121 Suppl 3:S192-207.

12. McLachlan JA. Environmental signaling: what embryos and evolution teach us about endocrine disrupting chemicals. Endocr Rev. 2001;22:319-41.

13. Rasier G, Toppari J, Parent AS, Bourguignon JP. Female sexual maturation and reproduction after prepubertal exposure to estrogens and endocrine disrupting chemicals: a review of rodent and human data. Mol Cell Endocrinol. 2006;254-255: 187-201.

14. Shea KM; American Academy of Pediatrics Committee on Environmental Health. Pediatric exposure and potential toxicity of phthalate plasticizers. Pediatrics. 2003;111:1467-74.

15. Hauser R, Calafat AM. Phthalates and human health. Occup Environ Med. 2005;62:806-18.

16. Latini G. Monitoring phthalate exposure in humans. Clin Chim Acta. 2005;361:20-9.

17. Swan SH. Environmental phthalate exposure in relation to reproductive outcomes and other health endpoints in humans. Environ Res. 2008;108:177-84.

18. Matsumoto M, Hirata-Koizumi M, Ema M. Potential adverse effects of phthalic acid esters on human health: a review of recent studies on reproduction. Regul Toxicol Pharmacol. 2008;50:37-49.

19. Heudorf U, Mersch-Sundermann V, Angerer J. Phthalates: toxicology and exposure. Int J Hyg Environ Health. 2007;210: 623-34.

20. Lyche JL, Gutleb AC, Bergman A, Eriksen GS, Murk AJ, Ropstad E, et al. Reproductive and developmental toxicity of phthalates. J Toxicol Environ Health B Crit Rev. 2009;12: 225-49.

21. Mouritsen A, Frederiksen H, Sørensen K, Aksglaede L, Hagen C, Skakkebaek NE, et al. Urinary phthalates from 168 girls and boys measured twice a year during a 5-year period: associations with adrenal androgen levels and puberty. J Clin Endocrinol Metab. 2013;98:3755-64. 
22. Durmaz E, Ozmert EN, Erkekoglu P, Giray B, Derman O, Hincal F, et al. Plasma phthalate levels in pubertal gynecomastia. Pediatrics. 2010;125:e122-9.

23. Frederiksen H, Sørensen K, Mouritsen A, Aksglaede L, Hagen $\mathrm{CP}$, Petersen $\mathrm{JH}$, et al. High urinary phthalate concentration associated with delayed pubarche in girls. Int J Androl. 2012;35: 216-26.

24. Wolff MS, Teitelbaum SL, Pinney SM, Windham G, Liao L, Biro $F$, et al. Investigation of relationships between urinary biomarkers of phytoestrogens, phthalates, and phenols and pubertal stages in girls. Environ Health Perspect. 2010;118: 1039-46.

25. Colón I, Caro D, Bourdony CJ, Rosario O. Identification of phthalate esters in the serum of young Puerto Rican girls with premature breast development. Environ Health Perspect. 2000; 108:895-900.

26. Chou YY, Huang PC, Lee CC, Wu MH, Lin SJ. Phthalate exposure in girls during early puberty. J Pediatr Endocrinol Metab. 2009;22:69-77.

27. Lomenick JP, Calafat AM, Melguizo Castro MS, Mier R, Stenger P, Foster MB, et al. Phthalate exposure and precocious puberty in females. J Pediatr. 2010;156:221-5.

28. Lee PA, Guo SS, Kulin HE. Age of puberty: data from the United States of America. APMIS. 2001;109:81-8.

29. Gelbrich G, Blüher S, Reich A, Müller G, Kiess W. Prevalence of obesity and elevated blood pressure as well as onset of puberty in German school children attending different educational tracks. Horm Res. 2008;70:340-8.

30. Sun Y, Tao FB, Su PY, Mai JC, Shi HJ, Han YT, et al. National estimates of the pubertal milestones among urban and rural Chinese girls. J Adolesc Health. 2012;51:279-84.

31. Sun Y, Tao F, Su PY; China Puberty Research Collaboration. National estimates of pubertal milestones among urban and rural Chinese boys. Ann Hum Biol. 2012;39:461-7.

32. de Onis M, Onyango AW, Van den Broeck J, Chumlea WC, Martorell R. Measurement and standardization protocols for anthropometry used in the construction of a new international growth reference. Food Nutr Bull. 2004;25(1 Suppl):S27-36.

33. Yao XJ, Liu CL, Chen Z, Zhang GY, Wang JH, Wang GL. A study on body fat in children aged 7-12. Chin J Prev Med. 1994;28:213-5.

34. Prader A. Testicular size: assessment and clinical importance. Triangle. 1966;7:240-3.

35. Ma HM, Chen SK, Chen RM, Zhu C, Xiong F, Li T, et al. Pubertal development timing in urban Chinese boys. Int $\mathrm{J}$ Androl. 2011;34:e435-45.

36. Marshall WA, Tanner JM. Variations in the pattern of pubertal changes in boys. Arch Dis Child. 1970;45:13-23.

37. Marshall WA, Tanner JM. Variations in pattern of pubertal changes in girls. Arch Dis Child. 1969;44:291-303.
38. Cochrane SH, Leslie J, O'Hara DJ. Parental education and child health - intracountry evidence. Health Policy Educ. 1982;2: 213-50.

39. Bradley RH, Corwyn RF. Socioeconomic status and child development. Annu Rev Psychol. 2002;53:371-99.

40. Kaplowitz PB. Link between body fat and the timing of puberty. Pediatrics. 2008;121 Suppl 3:S208-17.

41. Allison PD. Missing data, Sage University Papers Series on Quantitative Applications in the Social Sciences. CA: Sage: Thousand Oaks; 2001.

42. Wang H, Zhou Y, Tang C, He Y, Wu J, Chen Y, et al. Urinary phthalate metabolites are associated with body mass index and waist circumference in Chinese school children. PLoS One. 2013;8:e56800.

43. Qiao L, Zheng L, Cai D. [Study on the di-n-butyl phthalate and di-2-ethylhexyl phthalate level of girl serum related with precocious puberty in Shanghai]. Wei Sheng Yan Jiu. 2007; 36:93-5.

44. Mieritz MG, Frederiksen H, Sørensen K, Aksglaede L, Mouritsen A, Hagen CP, et al. Urinary phthalate excretion in 555 healthy Danish boys with and without pubertal gynaecomastia. Int J Androl. 2012;35:227-35.

45. Hannas BR, Lambright CS, Furr J, Howdeshell KL, Wilson VS, Gray LE Jr. Dose-response assessment of fetal testosterone production and gene expression levels in rat testes following in utero exposure to diethylhexyl phthalate, diisobutyl phthalate, diisoheptyl phthalate, and diisononyl phthalate. Toxicol Sci. 2011;123:206-16.

46. Huang PC, Kuo PL, Chou YY, Lin SJ, Lee CC. Association between prenatal exposure to phthalates and the health of newborns. Environ Int. 2009;35:14-20.

47. Swan SH, Main KM, Liu F, Stewart SL, Kruse RL, Calafat AM, et al. Decrease in anogenital distance among male infants with prenatal phthalate exposure. Environ Health Perspect. 2005;113: 1056-61.

48. Welsh M, Saunders PT, Fisken M, Scott HM, Hutchison GR, Smith LB, et al. Identification in rats of a programming window for reproductive tract masculinization, disruption of which leads to hypospadias and cryptorchidism. J Clin Invest. 2008;118: 1479-90.

49. Moore NP. The oestrogenic potential of the phthalate esters. Reprod Toxicol. 2000;14:183-92.

50. Hao C, Cheng X, Xia H, Ma X. The endocrine disruptor mono(2-ethylhexyl) phthalate promotes adipocyte differentiation and induces obesity in mice. Biosci Rep. 2012;32:619-29.

51. Bender R, Grouven U. Ordinal logistic regression in medical research. J R Coll Physicians Lond. 1997;31:546-51.

52. Gameroff M. Using the proportional odds model for healthrelated outcomes: Why, when, and how with various SAS procedures. Statistics and data analysis. SUGI. p. 205-30. 